\title{
SENTIDO DE VIDA, SAÚDE MENTAL E BEM-ESTAR EM ADULTOS: QUE RELAÇÕES?
}

\author{
Raquel Santos \\ Universidade Fernando Pessoa. Porto \\ raquel.santos@gdc.pt \\ Carla Fonte \\ Universidade Fernando Pessoa. Porto \\ cfonte@ufp.edu.pt \\ Cristina Pimentão \\ Universidade Fernando Pessoa. Porto \\ pimentão@ufp.edu.pt
}

Recepción Artículo: 14 diciembre 2020

Admisión Evaluación: 14 diciembre 2020

Informe Evaluador 1: 15 diciembre 2020

Informe Evaluador 2: 16 diciembre 2020

Aprobación Publicación: 17 diciembre 2020

\section{RESUMO}

Descobrir um sentido de vida é uma inquietação do ser humano que desde sempre existiu sendo considerado um objeto de estudo e foco de várias reflexões e teorias. Filósofos como Aristóteles e Epicuro afirmaram que o sentido da vida consiste em alcançar a verdadeira felicidade, reflectindo a necessidade de dar um significado à existência humana. Inicialmente, a investigação sobre o sentido da vida, ao tentar compreender a procura do indivíduo por um sentido, seguiu uma abordagem existencial, sendo que final do século XX, aquando da emergência da Psicologia Positiva emerge um maior investimento e conhecimento por parte da comunidade científica em torno destas questões, sendo o sentido da vida, considerado uma das componentes do bem-estar. Apresenta-se um estudo que teve como objectivo analisar a relação entre 0 sentido de vida, a saúde mental e o bem-estar numa amostra de 247 adultos, com idades compreendidas entre os 18 e os 69 anos. Os dados foram recolhidos com recurso ao Questionário de Sentido de Vida e à Escala Continuum de Saúde Mental. Os resultados indicaram que os indivíduos com níveis elevados de presença de sentido de vida apresentavam níveis igualmente elevados de bem-estar, enquanto que, por sua vez, os indivíduos com níveis elevados de procura de sentido de vida, apresentaram níveis menores de bemestar, sublinhando a forte relação entre estas duas dimensões.

Palavras-chave: sentido de vida; saúde mental; bem-estar 


\begin{abstract}
Life Meaning, Mental Health and Well-being in Adults: What Relationships? Discovering the meaning of life is a concern of the human being that has always existed, being considered an object of study and the focus of various reflections and theories. Philosophers like Aristotle and Epicurus stated that the meaning of life is to achieve true happiness, reflecting the need to give meaning to life is currently considered one of the components of mental health and well-being. In this context, a study is presented whose objective was to analyze the relationship of human existence. Initially the investigation of the meaning of life, when trying to understand the individual's search for a meaning, followed at an existential approach, and at the end of the 20th century, when the emergence of Positive Psychology emerges a greater investment and knowledge by the scientific community around these issues, the meaning of life hss being considered one of the components of well-being. A study is presented that aimed to analyze the relationship between the meaning of life, mental health and well-being in a sample of 247 adults, aged between 18 and 69 years. Data were collected using the Meaning of Life Questionnaire and the Mental Health Continuum Scale. The results indicated that individuals with high levels of presence of meaning of life had equally high levels of wellbeing, while, in turn, individuals with high levels of searging for life meaning, had lower levels of well-being, underlining the strong relationship between these two dimensions.
\end{abstract}

Keywords: life meaning; mental health; well-being

\title{
INTRODUÇÃO
}

A procura da felicidade e de uma vida com significado são, provavelmente, dois dos objetivos ancestrais que fazem parte da existência humana (Baumeister, Vohs, Aaker, \& Garbinsky, 2013). 0 homem procurou, desde os tempos primitivos, construir o sentido para a sua razão de ser e de estar no mundo através, por exemplo, da sua luta pela sobrevivência, com a descoberta e ocupação dos territórios. Ou seja, foi aos poucos tomando consciência da sua singularidade e condição diferenciada das outras espécies e, cada vez mais, desenvolveu meios para afirmar a sua presença no mundo e dar sentido à mesma (Carneiro \&Abritta, 2008). Há já vários anos que as raízes da reflexão filosófica sobre o sentido de vida fazem, a par da felicidade e do bem-estar, o objetivo de estudo da Psicologia (Kraus, Rodrigues, \& Dixe, 2008). Nos anos 90, com a emergência da Psicologia Positiva alicerça-se um forte investimento científico no estudo da felicidade, mas também sobre 0 que torna a vida significativa (Baumaister et al., 2013). Contudo, foi muitos anos antes com Vicktor Frankl, um psiquiatra e neurologista austríaco, e com o seu livro "0 Homem em busca de um sentido", que a temática do sentido de vida emergiu. Na sua obra, o autor descreve a sua vivência num campo de concentração em Auschwitz, relatando as suas teorias sobre o sentido da vida, descrevendo que a busca do indivíduo por um sentido para a sua vida é a sua motivação essencial e que esse sentido é então exclusivo e específico e que só pode ser cumprido por aquela pessoa (Frankl, 2012). Na sociedade atual, massificou-se a ideia de que ter um sentido na vida se relaciona com ter um bom emprego, uma casa, um carro, viajar, etc. Ou seja, os principais estímulos estão associados ao prazer e à distração e com isto, dar um sentido à vida, significa possuir determinado poder de compra (Lussier, 2000). Segundo Bastos (2004), o homem parece não saber o que fazer da sua vida demasiado materialista, em que 0 ter assumiu enormes proporções perante 0 ser, vivendo desta forma num conformismo típico da sociedade de consumo, em que a frustração existencial se instala por falta de interiorização e reflexão sobre o verdadeiro sentido da sua vida. A sensação de que a vida é vazia e inútil pode emergir, pela incapacidade de encontrar um sentido para a sua vida. Frankl (2012) refere que nenhum instinto diz ao ser humano aquilo que ele deve fazer e por isso ele acaba, por vezes, por fazer o que os outros fazem ou aquilo que os outros querem que ele faça, pois ele não sabe sequer o que deseja e a isto o autor chama de "vazio existencial", ou seja, inexistência 
de um sentido para a vida. Ao não sermos capazes de encontrar um sentido para a nossa vida, partindo das nossas crenças, valores e ideais pessoais, vivemos num estado de alienação permanente (Lussier, 2000). Assim, Frankl (2012), na sua obra, distingue várias formas de neurose e atribui algumas delas à incapacidade de encontrar um significado e um sentido de vida. A descoberta de um sentido de vida implica um autoconhecimento, em termos de valores, ideais, talentos, mas também de defeitos, carências e imperfeições. Só assim seremos capazes de criar e evoluir e delinear os objetivos que dão um sentido à nossa vida. Deste modo, para 0 autor, o sentido de vida exige autoconhecimento, compromisso, motivação, capacidade de encontrar beleza, principalmente nos maus momentos e ser capaz de extrair deles boas lições de resiliência. Emerge assim a ideia de que é importante ter a noção de que tudo aquilo que acontece pode ser positivo, se o mesmo for utilizado a nosso favor, de modo a atingir um melhor desenvolvimento pessoal e crescimento interior, sendo isto que leva a pessoa a encontrar o sentido da sua vida de uma forma harmoniosa e livre (Bastos, 2004).

Steger e Kashdan (2006) propuseram que o ser humano possui motivação para a presença e para a procura de sentido na vida. A presença de sentido na vida consiste na existência de um sentido. Já a procura de sentido na vida consiste no processo de busca e orientação pelo sentido. No entanto, esta procura pode estar relacionada com um estado de crise e/ou com formas negativas de enfrentamento (Dunn \& 0'Brien, 2009). Desta forma, existe uma relação inversa entre estas duas dimensões, sendo que a presença de sentido de vida se encontra positivamente relacionado com 0 bem-estar, enquanto, que a procura de sentido de vida se encontra relacionada ao bem-estar de forma negativa, pois quando não existe presença de sentido de vida, a procura dele pode tornar-se um processo difícil e frustrante (Park, \& Peterson, 2010). No entanto, a ausência de sentido de vida deve levar os indivíduos a procurá-lo resultando na descoberta de um propósito e significado satisfatório nas suas vidas (Steger \& Kashdan, 2006). 0 sentido de vida pode assim relacionar-se de uma forma positiva com o bem-estar quando existem níveis elevados de presença de sentido de vida, pois desta forma, os indivíduos têm uma base que permite que a procura de significado adicional leve a encontrar fontes novas ou adicionais de significado, ou a desenvolver uma compreensão mais aprofundada daquilo que já torna as suas vidas significativas, tornando-se num processo de modificação e expansão (Parket al., 2010; Steger \& Kashdan, 2006).

Ao longo das nossas vidas, aquilo que as pessoas mais tentam preservar e cuidar é a sua saúde, sendo este um fenómeno com um forte componente social, psicológica, mental, espiritual e física. Segundo a Organização Mundial de Saúde (2002), a saúde mental, a saúde física e a social estão relacionadas, sendo que, à medida que cresce a compreensão da mesma torna-se cada vez mais evidente que a saúde mental é indispensável para o bem-estar geral dos indivíduos, como também das sociedades e até dos países. No entanto, o valor atribuído à saúde mental nem sempre é mesmo aquele que é atribuído à saúde física. A investigação tem vindo a demonstrar que a saúde mental não inclui apenas a ausência de uma doença mental, mas também a presença de algo positivo, isto é, a saúde mental positiva, entendendo-se esta por uma síndrome de sintomas e sentimentos positivos otimizando as funções positivas na nossa vida. Esta definição assenta ainda em três componentes principais, o bem-estar emocional, o bem-estar psicológico e o bem-estar social que no seu conjunto integram a definição de saúde mental positiva (Keyes, 2002). Este autor propõe que a saúde mental positiva seja conceptualizada como um continuum, isto é, um estado completo em que os indivíduos estão livres de psicopatologia em que num extremo, se situa o estado de flourishing e no outro 0 estado de languishing. No meio destes encontraremos a saúde mental moderada. 0 estado de flourishing consiste numa combinação de bem-estar subjetivo com um nível ótimo de funcionamento psicológico e social. Já o estado de languishing é um estado de saúde mental no 


\section{SENTIDO DE VIDA, SAÚdE MENTAL E BEM-ESTAR EM ADULTOS: QUE RELAÇÕES?}

qual o indivíduo apresenta baixos níveis de bem-estar, ou seja, ausência de saúde mental. Neste âmbito, a presença de saúde mental positiva é considerada como flourishing e a ausência da mesma como languishing e aqueles que não se encontram em flourishing nem em languishing, são considerados com saúde mental moderada (Westerhof\&Keyes, 2010).

Paralelamente a esta conceptualização outros autores têm vindo a propor na conceptualização e compreensão do bem-estar, duas perspetivas distintas, mas complementares: a hedônica e a eudaimónica. Na primeira, o estudo do bem-estar perspetiva o bem-estar subjetivo, também designado como bem-estar hedónico ou emocional, ou seja, o equilíbrio favorável às emoções, aos sentimentos e afetos positivos sobre os negativos (Ryan \& Deci, 2000). Assim sendo, um bem-estar subjetivo elevado inclui frequentes experiências emocionais positivas, raras experiências emocionais negativas e satisfação não só com vários aspetos da vida, mas com a vida como um todo (Albuquerque \&Tróccoli, 2004). Já o bem-estar social refere-se à ligação entre as necessidades individuais e as necessidades sociais dos indivíduos (Zubieta \& Delfino, 2010), fornecendo uma avaliação subjetiva do funcionamento ideal de uma comunidade (Westerhof \& Keyes, 2010). Keyes (1998) propôs cinco dimensões do bem-estar social e que são a integração social, a aceitação social, a contribuição social, a atualização social ea coerência social.0 bem-estar psicológico é definido como a avaliação subjetiva do funcionamento individual e ideal. Ryff (1989) examinou várias teorias psicológicas acerca do desenvolvimento ideal de vida e sobre a saúde mental positiva, descobrindo seis elementos essenciais para o funcionamento positivo de um indivíduo subsequentemente analisadas e reformulados por Ryff e Keyes (1995). Em conjunto, compõem aquilo a que ele chama de bemestar psicológico, uma vez que cada um desses elementos é fundamental para a compreensão do potencial de cada um e para se tornar uma pessoa melhor, focando, principalmente o funcionamento ideal em termos de realização individual. Esses elementos são a autoaceitação, a autonomia, o crescimento pessoal, o domínio ambiental, as relações positivas com os outros e o sentido e propósito de vida.

Desde 0 início da nossa existência que o homem procura encontrar um sentido para a sua vida com 0 objetivo de atingir o bem-estar e a felicidade (Baumeisteret al., 2013; Bastos, 2004). Alguns estudos têm vindo a demonstrar a existência de uma relação entre o bem-estar e 0 sentido de vida, sendo que, segundo os mesmos, a presença de sentido de vida encontra-se sempre associada a um melhor bem-estar e à presença de saúde mental positiva (Brassaiet al., 2011; Steger \& Kawabata, Shimai \& Otake, 2007). Assim sendo, a presença de sentido de vida, poderá ser vista como uma componente, uma causa ou um resultado do bem-estar e como um fator de proteção para a saúde mental (Brassaiet al.,2011).

Atendendo a este cenário, desenvolveu-se um estudo com o objetivo de analisar a relação entre 0 sentido de vida, a saúde mental e o bem-estar em adultos. Mais concretamente, pretendemos caracterizar a amostra de estudo em função da existência de sentido de vida, a procura de sentido de vida ea saúde mental/bem-estar; analisar as diferenças do sentido de vida, saúde mental e bemestar em função de diferentes dimensões sociodemográficas; identificar a relação entre 0 sentido de vida experienciado pelos indivíduos e os níveis de saúde mental e bem-estar eidentificar as diferenças entre a presença e a procura de sentido de vida de acordo com os estados de saúde mental.

\section{MÉTODO}

\section{Participantes}

Neste estudo participaram 247 adultos, 67 do sexo masculino e 180 do sexo feminino, com idades compreendidas entre os 18 e os 69 anos de idade e uma média de idades de 36,9 anos. A maioria dos participantes são solteiros e casados (percentagem de $38,5 \%$ para ambos os casos), $14,2 \%$ 
estão em união de facto e $8,9 \%$ estão divorciados/separados ou viúvos. Relativamente às habilitações académicas, 75,7\%dos participantes tem formação superior, $21,1 \% 0$ ensino secundário e $3,2 \%$ onsino básico. A maioria dos participantes não tem filhos (53,4\%) e entre os $46,6 \%$ que têm filhos, 22,3\% tem apenas um, 17,8\%têm dois filhos, 4,0\% têm três filhos e 2,4\% quatro filhos. Todos os participantes residem em Portugal.

\section{Instrumentos}

Neste estudo utilizamos os seguintes instrumentos para a recolha de informação:

Questionário Sociodemográfico - Este questionário teve como objetivo recolher dados que permitissem uma adequada caracterização da amostra em função das variáveis a analisar: sexo, idade, habilitações literárias, estado civil, descendência e número de filhos.

Questionário do Sentido de Vida - Versão Portuguesa - 0 Questionário do Sentido de Vida (QSV) (Portugal, 2017) versão portuguesa do The Meaning in Life Questionnaire (Steger \& Kashdan, 2006) é composto por duas subescalas de cinco itens cada, com o objetivo de medir a presença e a procura de sentido de vida. A subescala presença de sentido de vida, tem como objetivo detetar a existência de sentido da vida e é composta por cinco itens:1; 4; 5; 6 e 9 (ex.: item 1. "Eu compreendo 0 sentido da minha vida."). A subescala procura, também composta por cinco itens: 2; 3; 7; 8 e 10 (ex.: item 8 "Estou à procura de um sentido ou missão para a minha vida.") permite avaliar a procura e o processo de busca de sentido da vida. Cada item é avaliado através de uma escala de Likert de 7 pontos, em que 1 corresponde a "Absolutamente Falso" e 7 a "Absolutamente Verdadeiro". A cotação do instrumento exige a inversão do item 9.

Escala Continuum de Saúde Mental (MHC-SF) - Versão Portuguesa - Esta escala foi adaptada para Portugal por Fonte, Silva, Vilhena, e Keyes (2020) e avalia as três dimensões da saúde mental positiva: bem-estar emocional/subjetivo, bem-estar social, e bem-estar psicológico, num total de 14 itens: três itens para o bem-estar emocional (itens 1-3), cinco itens para o bem-estar social (itens 4-8) e seis itens para o bem-estar psicológico (itens 9-14). Esta escala permite a caracterização dos indivíduos em três categorias: flourishing, languishing e saúde mental moderada, numa escala tipo Likert de 6 pontos, em que 1 representa "nunca" e 6 "todos os dias". Para ser classificado no estado de flourishing os indivíduos têm de pontuar 5 ou 6 pontos (quase todos os dias; todos os dias) em pelo menos um dos três itens correspondentes ao bem-estar emocional/subjetivo e em pelo menos seis dos onze itens de bem-estar social e psicológico. Para ser classificado no estado de languishing são necessárias pontuações baixas em pelo menos um dos três itens do bem-estar emocional e em pelo menos seis dos onze itens do bem-estar social e psicológico (entende-se por pontuações baixas 'nunca' e 'uma ou duas vezes por semana'). 0 diagnóstico de saúde mental moderada é atribuído quando se pontua 3 ou 4 ('cerca de uma ou duas vezes por semana'; 'cerca de duas ou três vezes por semana') em pelo menos um dos três itens do bem-estar emocional e em pelo menos seis dos onze itens do bem-estar social e psicológico (Fonte et al., 2020; Keyes, 2005). Em relação aos scores totais, para 0 total da escala e para cada dimensão, os valores encontram-se entre: para o total da escala 15-50; para o bem-estar emocional3-18; para 0 bem-estar social5-30; e para o bem-estar psicológico7-42 (Fonte et al., 2020).

\section{Procedimento}

Este estudo faz parte de um projeto de investigação mais alargado intitulado "A.Life.Meaning LifeMeaning, mental health, well-being and work: what relationships?". 0 projeto foi submetido e aprovado pela Comissão de Ética da Universidade do Norte de Portugal, Porto, onde o projeto de investigação foi proposto. A recolha de dados foi efetuada online e de forma a informar e esclarecer os participantes sobre 0 estudo e o conteúdo dos instrumentos, salvaguardando 0 anonimato e a 


\section{SENTIDO DE VIDA, SAÚDE MENTAL E BEM-ESTAR EM ADULTOS: QUE RELAÇÕES?}

confidencialidade, foi numa primeira fase, obtida a autorização dos participantes (assentimento informado), sendo este um requisito obrigatório para aceder aos conteúdos do questionário. Todos os instrumentos estiveram disponíveis durante o tempo de resposta através de um link, criado através do Google Forms,que foi acedido pelos participantes. A recolha de dados foi feitade forma nãoaleatória, pelo método de Snowball, com a divulgação do link do estudo através da rede de contactos dos investigadores. 0 tratamento estatístico dos dados foi realizado através do programa Statistical Package for the Social Sciences (SPSS), versão 25, sendo utilizadas análises descritivas, o Teste de Associação- Coeficiente de correlação de Pearson e Teste de Diferenças em contexto de Design Intersujeitos, nomeadamente o Teste T para Amostras Independentes, a Análise de variância (ANOVA) Unifatorial.

\section{RESULTADOS E DISCUSSÃO}

\section{Níveis de sentido de vida e saúde mental positiva/bem-estar}

Os participantes revelaram níveis elevados de presença e de procura de sentido de vida, com preponderância para a presença de sentido de vida (Tabela 1). Estes resultados vão de encontro à literatura consultada, sendo que, segundo os autores, como existem níveis elevados de presença de sentido de vida, os indivíduos revelam também níveis elevados de procura, no sentido de desenvolver uma compreensão mais aprofundada daquilo que já dá sentido as suas vidas ou de procurar acrescentar novas fontes de significado, tornando-se um processo de modificação e de expansão (Parket al, 2010; Steger \& Kashdan, 2006).

Tabela 1

Médias, desvios-padrão e valores máximos e mínimos, em função da presença de sentido de vida e de procura de sentido de vida

\begin{tabular}{|c|c|c|c|c|}
\hline \multicolumn{5}{|c|}{$\operatorname{Amostra}(N=247)$} \\
\hline & Mínimo & Máximo & Média & $\begin{array}{l}\text { Desvio } \\
\text { Padrão }\end{array}$ \\
\hline Presença de sentido de vida & 6 & 35 & 27.55 & 5.53 \\
\hline Procura de sentido de vida & 5 & 35 & 19.19 & 8.07 \\
\hline
\end{tabular}

A maioria dos participantes apresentaram níveis elevados de bem-estar, atendendo aos valores mínimos e máximos (Tabela 2).

Tabela 2

Médias, desvios-padrão e valores máximos e mínimos, em função do bem-estar geral, bem-estar emocional, bem-estar social e bem-estar psicológico

\begin{tabular}{|c|c|c|c|c|}
\hline \multicolumn{5}{|c|}{$\operatorname{Amostra}(N=247)$} \\
\hline & Mínimo & Máximo & Média & $\begin{array}{l}\text { Desvio } \\
\text { Padrão }\end{array}$ \\
\hline Bem-estar emocional & 3.00 & 18.00 & 14.44 & 2.85 \\
\hline Bem-estar social & 5.00 & 30.00 & 19.22 & 5.40 \\
\hline Bem-estar psicológico & 6.00 & 36.00 & 29.08 & 5.65 \\
\hline Bem-estar total & 14.00 & 84.00 & 62.74 & 12.47 \\
\hline
\end{tabular}


Constatou-se ainda que a maioria dos participantes se encontra em flourishing (63.6\%), seguindo-se os que se encontram com saúde mental moderada (32.8\%) eos que se encontram em languishing (Tabela 3).

Tabela 3

Níveis de saúde mental dos participantes em termos de saúde mental positiva/bem-estar

\begin{tabular}{lcc}
\hline & Amostra $(\boldsymbol{N}=\mathbf{2 4 7})$ & \\
\hline & $\boldsymbol{N}$ & $\boldsymbol{\%}$ \\
\cline { 2 - 3 } Flourishing & 157 & 63.6 \\
Saúde mental moderada & 81 & 32.8 \\
Languishing & 9 & 3.6 \\
\hline
\end{tabular}

\section{Sentido de vida, saúde mental e bem-estar em função de diferentes dimensões sociodemográ-} ficas

Estado civil - Verificaram-se diferenças, estatisticamente, significativas entre os casados e os solteiros na procura de sentido de vida $(f=4.21, p=.006)$, apresentando os casados uma média inferior na procura de sentido de vida quando comparados com os solteiros. Foram encontradas diferenças significativas ao nível do bem-estar emocional ( $f=3.02, p=.031)$ entre os solteiros e os separados/divorciados/viúvos ( $f=3.02, p=.024$ ) e entre os casados e os separados/divorciados/viúvos ( $f=3.02, p=.031$ ), verificando-se, através do teste Post-Hoc de Bonferroni, que os separados/divorciados/viúvos apresentam menores níveis de bem-estar emocional do que os solteiros e os casados. Estes resultados encontram eco em pesquisas que apontam para o facto de o casamento estar fortemente associado ao bem-estar subjetivo/emocional, sendo que ter um conjugue representa uma fonte de suporte social e por isso é normal que esteja relacionado com o aumento dos níveis de bem-estar subjetivo nos indivíduos. As pessoas casadas ou numa relação são, em média, mais felizes, têm uma melhor saúde mental e uma maior satisfação com a vida, do que as solteiras, viúvas ou divorciadas (Argyle, 1999; Diener et al., 2000; Haring-Hidore, 1985).

Habilitações académicas - Encontraram-se diferenças, estatisticamente, significativas ( $f=3.16$, $p=.04)$ ao nível do bem-estar social. 0 teste Post-Hoc de Bonferroni revelou que os participantes com o ensino básico revelam níveis menores de bem-estar social, do que os indivíduos com o ensino superior.

Filhos - Os participantes que têm filhos relataram níveis significativamente inferiores de procura de sentido de vida ( $t=-3.47, p=.001)$. Estes resultados estão de acordo com os encontrados na literatura que apontam para que a procura de um sentido resulte na descoberta de um propósito, satisfatório, para a vida, sendo que a existência de filhos poderá contribuir ou constituir-se na atribuição de um sentido, satisfatório, para a vida dos pais (Steger \& Kashdan, 2006).

Idade - Verificou-se uma correlação negativa e significativa entre a procura de sentido de vida e a idade ( $r=-.150, p=.018)$ que indica que quanto maior a idade dos indivíduos, menor é a sua procura de sentido de vida. Estudos apontam para que os mais novos tendam a olhar mais para o futuro e para a procura de novos objetivos, enquanto que os mais velhos têm tendência a olhar mais para o seu passado e para aquilo que já alcançaram nas suas vidas (Maia, 2015).

\section{Relação entre sentido de vida e níveis de saúde mental positiva/bem-estar}

Constatou-se a existência de uma correlação negativa e estatisticamente significativa entre a procura de sentido de vida e todas as restantes dimensões em análise neste estudo. Isto é, com a presença de sentido de vida $(r=-.225, p=.000)$, com 0 bem-estar total $(r=-.217, p=.001)$, com 0 


\section{SENTIDO DE VIDA, SAÚDE MENTAL E BEM-ESTAR EM ADULTOS: QUE RELAÇÕES?}

bem-estar emocional $(r=-.220, p=.001)$, com 0 bem-estar social $(r=-.133, p=.037)$ e com 0 bemestar psicológico ( $r=-.241, p=.000$ ) (Tabela 4). Estes resultados mostram que quanto maior for a procura de sentido de vida, menor será a presença de sentido de vida, e de bem-estar geral, emocional, psicológico, e social. Em relação à presença de sentido de vida, podemos verificar uma correlação estatisticamente significativa com 0 bem-estar geral $(r=.631, p=.000)$, 0 bem-estar emocional $(r=.617, p=.000)$, 0 bem-estar social $(r=.426, p=.000)$ e 0 bem-estar psicológico $(r=-.674$, $p=.000$ ) (Tabela 4). Estes resultados demonstram que os participantes com níveis elevados de presença de sentido de vida, evidenciam, também, níveis elevados de bem-estar geral, bem-estar emocional, bem-estar social, e bem-estar psicológico e, consequentemente, níveis mais baixos de procura de sentido de vida. Estudos evidenciam que à medida que os níveis de presença de sentido de vida aumentam, a sua procura diminui e consequentemente os níveis de felicidade também aumentam (Cohen \& Cairns, 2011; Hallfor et al, 2016; Parket al., 2010). Quanto às dimensões do bemestar, para além da correlação negativa estatisticamente significativa com a procura de sentido de vida e com a correlação estatisticamente significativa com a presença de sentido de vida, estas dimensões influenciam-se de uma forma positiva entre si. Foi possível constatar que quanto maiores os níveis de bem-estar em geral, maiores os níveis dos participantes com o bem-estar emocional $(r=.852, p=.000)$, o bem-estar social $(r=.897, p=.000)$ e com 0 bem-estar psicológico $(r=.918$, $p=.000$ ) (cf. Tabela 4). Em relação ao bem-estar emocional, quanto mais elevado o nível de bemestar emocional dos participantes, maior 0 nível de bem-estar social $(r=.670, p=.000)$ e de bemestar psicológico ( $r=734, p=.000$ ) (Tabela 4). Quanto maior o nível de bem-estar social, maior o nível de bem-estar psicológico $(r=.684, p=.000)$ e vice-versa. Ter um significado e propósito de vida é essencial para a saúde mental e o bem-estar (Cohen \& Cairns, 2011; Hallfordet al. 2016; Parket al., 2010).

Tabela 4

Correlações entre sentido de vida e os níveis de saúde mental e bem-estar

\begin{tabular}{lcccccc}
\hline \multicolumn{7}{c}{ Amostra $(N=247)$} \\
\hline & $\begin{array}{c}\text { Presença } \\
\text { de } \\
\text { sentido } \\
\text { de vida }\end{array}$ & $\begin{array}{c}\text { Procura } \\
\text { de sentido } \\
\text { de vida }\end{array}$ & $\begin{array}{c}\text { Bem-estar } \\
\text { total }\end{array}$ & $\begin{array}{c}\text { Bem-estar } \\
\text { emocional }\end{array}$ & $\begin{array}{c}\text { Bem-estar } \\
\text { social }\end{array}$ & $\begin{array}{c}\text { Bem-estar } \\
\text { psicológico }\end{array}$ \\
\hline $\begin{array}{l}\text { Presença de } \\
\text { sentido de vida }\end{array}$ & 1 & $-.225^{* *}$ & $.631^{* *}$ & $.617^{* *}$ & $.426^{* *}$ & $.674^{* *}$ \\
\hline $\begin{array}{l}\text { Procura de } \\
\text { sentido de vida }\end{array}$ & $-.225^{* *}$ & 1 & $-.217^{* *}$ & $-.220^{* *}$ & $-.133^{*}$ & $-.241^{* *}$ \\
\hline $\begin{array}{l}\text { Bem-estar } \\
\text { total }\end{array}$ & $.631^{* *}$ & $-.217^{* *}$ & 1 & $852^{* *}$ & $897^{* *}$ & $.918^{* *}$ \\
\hline $\begin{array}{l}\text { Bem-estar } \\
\text { emocional }\end{array}$ & .617 & $-.220^{* *}$ & $.918^{* *}$ & 1 & $.670^{* *}$ & $.734^{* *}$ \\
\hline $\begin{array}{l}\text { Bem-estar } \\
\text { social }\end{array}$ & $.426^{* *}$ & $-.133^{*}$ & $.897^{* *}$ & $.670^{* *}$ & 1 & $.684^{* *}$ \\
\hline $\begin{array}{l}\text { Bem-estar } \\
\text { psicológico }\end{array}$ & $.674^{* *}$ & $-.241^{* *}$ & $.852^{* *}$ & $.734^{* *}$ & $.684^{* *}$ & 1 \\
\hline$* p<.05 * * p<0.01$ & & & & & \\
\hline
\end{tabular}

Os participantes que se encontravam em flourishing apresentaram níveis mais elevados de presença de sentido de vida e níveis menores de procura de sentido de vida, enquanto que os que esta- 
vam em languishing apresentaram níveis mais elevados de procura de sentido de vida e menores níveis de presença de sentido de vida. Estes resultados corroboram os encontrados noutros estudos que mostram que quando os indivíduos consideram a sua vida significativa, com presença de sentido de vida, também apresentam níveis mais elevados de bem-estar e satisfação com a vida (flourishing).

\section{CONCLUSÕES}

A investigação tem evidenciado, ao longo do tempo, que a saúde mental para além da ausência de doença mental envolve, também, a presença de algo positivo na vida. Tal facto traduz-se na saúde mental positiva, envolvendo aspetos emocionais, psicológicos e sociais que permitem observar a saúde como um estado completo. Neste âmbito, diversos trabalhos de investigação têm vindo a apontar para a existência de uma relação entre o bem-estar e 0 sentido de vida. Este estudo teve como objetivo principal aprofundar o conhecimento sobre a relação entre o sentido de vida, a saúde mental e 0 bem-estar, em adultos.0s resultados mostraram que a maioria dos participantes se encontrava em flourishing (uma combinação de bem-estar subjetivo com um nível ótimo de funcionamento psicológico e social) e que apresentavam níveis elevados de presença e de procura de sentido de vida. Foram os indivíduos mais velhos e os casados que apresentaram níveis menores de procura de sentido de vida. Em relação aos níveis de bem-estar, relativamente ao bem-estar emocional, os indivíduos separados/divorciados/viúvos foram os que apresentaram menores níveis. Quanto ao bem-estar social, foram os participantes com o ensino básico que apresentaram níveis menores.Quanto à relação entre o sentido de vida experienciado pelos indivíduos e os níveis de saúde mental e bem-estar, no que respeita à presença e à procura de sentido de vida, constatou-se que, os participantes com níveis mais elevados de presença de sentido de vida, de bem-estar geral, bem-estar emocional, bem-estar social e bem-estar psicológico evidenciaram níveis menores de procura de sentido de vida. Verificou-se ainda, que, os participantes com níveis elevados de presença de sentido de vida evidenciaram também níveis elevados de bem-estar geral, bem-estar emocional, bem-estar social e de bem-estar psicológico, ou que, participantes com níveis elevados de bem-estar geral, bem-estar emocional, bem-estar social e bem-estar psicológico revelaram também níveis elevados de presença de sentido de vida. Em relação às dimensões do bem-estar foi possível constatar que, quanto maiores os níveis de bem-estar em geral, maiores os níveis dos participantes com 0 bem-estar emocional, bem-estar social e bem-estar psicológico. Por último, na análise do estudo da relação entre a procura de sentido de vida e os estados de saúde mental, foi possível verificar-se que os indivíduos que se encontravam em flourishing apresentaram níveis mais elevados de presença de sentido de vida e níveis menores de procura de sentido de vida e que, já os indivíduos em languishing apresentaram níveis mais elevados de procura de sentido de vida e menores níveis de presença de sentido de vida. 0 presente estudo contribuiu assim para a compreensão da existência de uma relação entre 0 sentido de vida, a saúde mental e o bem-estar, contribuindo também, para 0 aumento do conhecimento científico sobre estas variáveis e sobretudo sobre a forma como elas se relacionam. Chegou a hora de considerar novas perspetivas sobre os fatores que influenciam o bem-estar. Propomos que a busca e descoberta de um sentido seja um processo de desenvolvimento pessoal, de conscientização, de sincronização com o verdadeiro eu e de viver uma vida significativa. De facto, o bem-estar humano tem sido um foco central das ciências sociais que persistiu na tarefa de descobrir por que as pessoas são infelizes. Mas, nas últimas décadas, o desafio de encontrar rotas para a felicidade humana tomou um novo rumo, com um foco explícito não apenas nas causas do sofrimento, mas também nas origens dos estados positivos de bem-estar. Essas abordagens integram os componentes e sistemas que podem influenciar o bem-estar, como eventos da vida, relacionamentos, personalidade, fatores culturais ou fatores biológicos, sugerindo que 


\section{SENTIDO DE VIDA, SAÚDE MENTAL E BEM-ESTAR EM ADULTOS: QUE RELAÇÕES?}

0 efeito positivo e o negativo são um continuum - não fins opostos (Westerhof \& Keyes 2010). Embora a maioria das pessoas pense que a vida normal é feliz e outros estados sejam anormalidades, os seres humanos são projetados para os dois estados: felicidade e infelicidade. Assim os dados deste estudo permitem propor que, no mundo moderno devemos aceitar as condições de que felicidade e infelicidade são meios para alcançar o significado da vida. 0 bem-estar é mais que emoções agradáveis, é uma condição positiva e sustentável que engloba a resiliência humana, porque sempre haverá contratempos inerentes ao processo de viver. Concordamos assim com a ideia de que 0 sentido da vida, como Viktor Frankl propôs (2012), pode melhorar a capacidade de desenvolver e prosperar diante das adversidades da vida e é um elemento-chave do bem-estar. Em momentos de dificuldade, as pessoas podem adquirir um novo senso de propósito, desenvolver relacionamentos mais profundos, apreciar melhor a vida e relatar outros benefícios. Isto é a capacidade humana de transformar criativamente os aspetos negativos da vida em algo positivo ou construtivo.

\section{REFERÊNCIAS BIBLIOGRÁFICAS}

Albuquerque, A. \& Tróccoli, B. (2004). Desenvolvimento de uma escala de bem-estar subjetivo. Psicologia: teoria e prática, 20 (2), 153-164.

Baumeister, R. F., Vohs, K. D., Aaker, J. L., \&Garbinsky, E. L. (2013). Some key differences between a happy life and a meaningful life. TheJournalof Positive Psychology, 1, 1-12.

Bastos, A. (2004). Um sentido para a vida- 0 equilíbrio do infinito do ser. Águeda: Paulinoseditora.

Brassai, L., Piko, B., \& Steger, M. (2011). Meaning in Life: Is It a Protective Factor for Adolescents' Psychological Health? InternationalSocietyofBehavioral Medicine, 18,44-51.

Carneiro, C., \&Abritta, S. (2008). Formas de existir: A busca de sentido para a vida. Revista de Abordagem Gestáltica, 14, 190-194.

Cohen, K., \& Cairns, D. (2011). Is searching for meaning in life associated with reduced subjective well-being? Confirmation and possible moderators. Journal of happiness studies, 13 (2), 313331.

Dunn, M. G., \& O'Brien, K. M. (2009). Psychological Health and Meaning in Life Hispanic. JournalofBehavioralSciences, 31(2), 204-22.

Frankl, V. (2012). O homem em busca de um sentido. Alfragide: Lua de papel.

Fonte, C., Silva, I., Vilhena, E. \& Keyes, C. (2020). The Portuguese Adaptation of the Mental Health Continuum-Short Form for Adult Population. Community Mental Health Journal, 56 (2), 368375, DOI 10.1007/s10597-019-00484-8.

Hallford, D. J., Mellor, D., Cummins, R. A., \& McCabe, M. P. (2016). Meaning in Life in Earlier and Later Older-Adulthood: Confirmatory Factor Analysis and Correlates of the Meaning in Life Questionnaire. Journal of Applied Gerontology, 3, 72 -73.

Haring-Hidore, M., Stock, W. A., Okun, M. A., \& Witter, R. A. (1985). Marital Status and Subjective Well-Being: A Research Synthesis. Journal of Marriage and the Family, 47(4), 947.

Keyes, C. (1998). Social well-being. Social Psychology Quarterly, 61 (2), 121-140.

Keyes, C. (2002). The mental health continuum: from languishing to flourishing in life. Journal of Health and Social Research, 43, 207-222.

Keyes, C. (2005). Mental illness and/or mental health? Investigating axioms of the complete state model of health. Journal of Consulting and Clinical Psychology, 73(3), 539-548

Kraus, T., Rodrigues, M., \& Dixe, M. (2008). Sentido de vida, saúde e desenvolvimento humano. Revista referência, 10, 77-88.

Lussier, M. (2000). Conhecer-se e melhor viver. Sintra: Publicações Europa-América. 
Maia, J. (2015). O bem-estar psicológico e satisfação com a vida em pessoas adultas e idosas. (Dissertação de Mestrado, Universidade dos Açores, Portugal).

Organização Mundial de Saúde. (2002). Relatório Mundial da Saúde-Saúde Mental: nova conceção, nova esperança. (1eed). Lisboa: Direção Geral da Saúde.

Park, N., Park, M., \& Peterson, C. (2010). When is the search for meaning related to life satisfaction? Jounalofoccupationalandorganizationalpsychology, 2 (1), 1-13.

Portugal, M. (2017). Versão portuguesa do questionário do sentido da vida: primeiros estudos psicométricos (Dissertação de Mestrado, Universidade de Lisboa, Lisboa, Portugal).

Ryan, R. M. \&Deci, E. L. (2001). On happiness and human potentials: a review of research on hedonic and eudaimonic well-being. Annual Review of Psychology, 52, 141-166

Ryff, C. (1989). Happiness is everything, or is it? Explorations on the meaning of psychological wellbeing. Journal of personality and social psychology, 57 (6), 1068-1081.

Ryff, C. \& Keyes, C. (1995). The structure of psychological well-being revisited. Journal of Personality and Social Psychology, 69 (4), 719-727

Steger, M., Kawabata, Y., Shimai, S. \& Otake, K. (2007). The meaningful life in Japan and the United States: levels and correlates of meaning in life. Journal of Research in Personality, 42, 660-678.

Steger, M. F., \&Kashdan, T. B. (2006). Stability and specificity of meaning in life and life satisfaction over one year. Journal of Happiness Studies, 8(2), 161-179.

Vivaldi, F.\& Barra, E. (2012). Bienestar psicológico, apoyo social percebido y percepción de salude en adultos mayores. Terapia Psicológica, 30 (2), 23-29.

Westerhof, G. J. \& Keyes, C. L. M. (2010). Mental illness and mental health: the two continua model across the lifespan. Journal of Adult Development, 17, 110-119.

Zubieta, E., Delfino, G. (2010). Satisfacción com la vida, bienestar psicológico y bienestar social en estudantesuniversitários de Buenos Aires. Faculdad de psicologia - UBA/ secretaria de investigaciones/ anuário de investigaciones, 17, 277-283. 
